\title{
Evaluating and choosing: the role of mathematics
}

\author{
Enrico Rogora
}

Published online: 4 February 2014

(c) Centro P.RI.ST.EM, Università Commerciale Luigi Bocconi 2014

\begin{abstract}
The use of numerical indexes and mathematical models is not automatically synonymous for objectivity, nor does it remove responsibility for the choices made. The paper points at some of the hazards of the confusion between using mathematics and objectivity and discusses the role of models in making rational choices.
\end{abstract}

\section{Keywords Psychometric models · Mathematical models}

When we have to make a choice, we can use either our hearts or our heads. When we use our heads, we try to evaluate the advantages and costs of the various options and predict their consequences. Using numbers and mathematical models for evaluating and predicting appears to be a good strategy for guaranteeing the objectivity of the choices and maximising the probability of making the best choice, but in reality this trust in numbers and mathematical models is not always merited, and the hope of evaluating objectively is often unfounded. This does not mean that mathematics in not a fundamental instrument for making rational choices, but that we must be aware of its limitations and of the risk of its being used to conceal responsibility or interests that are involved in those choices, instead of helping to clarify the terms of the problem in a rational manner.

\section{Numbers for evaluating}

Our choices often depend on numbers. For example: if my weight increases, I begin a diet; if the temperature is

E. Rogora $(\bowtie)$

Dipartimento di Matematica, Sapienza Università di Roma, Piazzale Aldo Moro, 5, 00185 Rome, Italy

e-mail: rogora@mat.uniroma1.it expected to go down, I wear warm clothes; if my scores on aptitude examinations are low in some subjects, I avoid choosing certain degree courses at university; if the difference in earnings (spread) between Italian and German government bonds goes up and the price of German bonds goes down, then the Italian government takes exceptional economic measurements. It is natural to look for simple criteria that help us to make a choice, and numerical criteria seems to be the simplest and most objective possible, but this isn't always the case. A measurement such as weight and temperature provides us with objective information regarding a well-defined property of an object, but the situation becomes more obscure when the numbers are not the result of a measurement or when the measurements are very imprecise and so indirect that it is not even possible to understand clearly what is being measured.

The number 300 is equal for everyone, but while $300 \mathrm{~kg}$ is an objective measure of weight, a spread of 300 points between 10-year Italian government bonds and analogous German bonds doesn't measure anything (see the appendix 'Fit to be tied: Manifesto of Appalled Economists'). It often happens that numbers and measurements are confused, and that no account is taken of the fact that measuring is more complex than assigning a number.

When we use an numerical index as though it were a measurement, we commit gross errors. For example, by now it must be clear to everyone that the spread is not capable of thoroughly measuring the healthiness of an economy, and that the very idea of measuring the state of the economy with a number is a dangerous stretch. Again, if we say that the students who have taken the 2012 entrance exam in medicine are smarter than those who took it in 2013 because the average number of correct answers is higher, then we are not taking into account the fact that the reason might be that the questions were easier. Further: is 
there really such a thing as ability that can be adequately weighed?

In the social sciences and the economy we cannot define direct measurements such as weight and length, or other measurements derived from physics. In these areas the most we can define - and with a great deal of caution-are indirect measurements that are estimated starting with the data, using mathematical models that hypothesise a link between the measurements and the observed data (see the appendix 'On statistical models for the analysis of tests'). It is always necessary, when indirect measurements are introduced, to make sure that the models used are in close agreement with the data, and that the hypotheses of the model are at least approximately verified. Otherwise the estimated measurements lose all meaning. This constitutes a serious risk for the transparency of the indirect measurements, for example, in the evaluations, because control of their quality and their significance is delegated, in the best case scenario, to a group of experts, and in the worst case to blind trust in automatic procedures.

A numerical index can always be used to create rankings. The point is that the classifications are not objective just because they are based on numerical indicators.

Having blind faith in numbers is not a rational attitude. What is necessary is a critical approach that question the significance and properties of numerical indexes and the faith that we place in mathematical models and indirect measurements that are drawn from them.

\section{Uses and abuses of mathematical models}

From weather forecasts to automated systems of flight controls, from the evaluation of risk of financial investments to the design of oil drilling rigs, from voice recognition systems to the voice analyses used by criminal investigators: these are just a few of the innumerable examples of the applications of mathematical models that have profound implications in everyday life. Questioning the uses and abuses of mathematical models and the faith that we have in their predictions is important for having a greater awareness of many fundamental issues that our future depends on: economic and financial policies (or lack of policies), environmental and energy policies (or lack of policies), and so forth.

There are multiple ways of using mathematical models. The most familiar is that of simulating the evolution of several magnitudes in order to plan (bridges, airplanes, oil rigs, etc.) or predict (atmospheric conditions, the behaviour of financial markets, energy needs, etc.). Also important are the applications for estimating hidden variables which we alluded to in the preceding section, for example, the use of models to estimate indirect measurements.
As the complexity of the reality to be modelled increases, a model is capable of providing descriptions that are increasingly less detailed, but no less useful because of this. It is necessary to renounce the predictability of the minute details of a phenomenon and limit ourselves to using the model to predict only some of the salient characteristics that, in a good model, are those that are of the greatest interest for planning an intervention or making decisions. Further, it is clear that predictions become less reliable the further into the future we try to see. Predictions based on mathematical models are increasingly conditioning our everyday life. How reliable are these predictions and up to what point is it wise to trust them? Already in the area of meteorological forecasts we know that it is possible for there to be significant discrepancies between what the model predicts and what happens in reality. For example, the prediction of bad weather in certain areas can lead to a big decrease in tourist reservations, with notable economic consequences that are not always justified by the weather conditions that actually occur. Even more macroscopic examples of the cost of erroneous predictions are found in the environmental sector, where the responses of markets to the predictions of the model have enormous repercussions for the economic situation (see again the appendix 'Fit to be tied: Manifesto of Appalled Economists').

It is true that in 1760 Daniel Bernoulli wrote (as an introduction to a mathematical model of his on the spread of smallpox): 'when decisions must be made that involve man's wellbeing, no decision could be taken without the knowledge that calculations can provide'. Bernoulli was not however suggesting the use of mathematics as a means of eliminating responsibility for the choices, but was rather simply suggesting that, whenever possible, the elements that emerge from the analysis of a mathematical model should be consulted in making responsible choices.

In many contexts, trusting the predictions of a model can involve elevated costs in coping with phenomena that turn out to be negligible. This was the case with the predictions of the spread of SARS in countries in the West, which caused huge sums to be spent for vaccines that went unused. These 'false alarms' (and perhaps, sometimes, 'procured alarms') have the consequence of justifying the downplaying of signals of alarm set off by other models, such as the exhaustion of planetary resources, or the nonsustainability of our economies. But, then, in which models' predictions should we believe, or does it make any sense at all to have faith in the predictions of a mathematical model?

The predictions of a mathematical model are not objective, but opinionable, and should be considered and discussed as opinions: rational, transparent, logical, at least for those who know more or less how the model 
functions, but always opinions and not absolute truths. The predictions of a mathematical model can be very helpful for clarifying the terms of the issues that must be faced, rendering the discussion more rational and the choices easier to agree upon, but they cannot and must not substitute the one who has to take responsibility for the choice. It must always be borne in mind that the use of a mathematical model can make a choice clearer if the general meaning of the model and its limitations are well understood; otherwise, they easily become instruments in the hands of those who wish to condition the choices. It is the duty of mathematicians and scientists to denounce the irresponsibility that is often observed in the use of numbers, mathematical models, statistics, etc., in the name of an ideal of objectivity that is profoundly anti-scientific. The choices cannot and must not be objective, but responsible and transparent. In Italy the choices are often manipulated and scarcely transparent, but the alternative of making them automatic by basing them on numerical indexes or the predictions of models that are little understood and badly adapted is a cure that is worse than the disease.

Translated from the Italian by Kim Williams.

\section{Appendix: Fit to be tied. Manifesto of Appalled Economists}

(http://www.atterres.org/page/manifeste-deconomistesatterres).

According to the proponents of efficient capital markets, market operators take into account the objective situation of public finances in order to assess the risk of taking out state bonds. Take the case of Greek debt: financial operators and policy makers rely exclusively on financial assessments in order to assess the situation. Thus, when the required interest rate for Greece rose to more than $10 \%$, everyone concluded that the risk of default was high: if investors demanded such a risk premium, this meant that the danger was extreme. This is a profound mistake if one understands the true nature of the assessment by the financial market. As this market is not efficient, it very often produces prices disconnected from the fundamentals. In these circumstances, it is unreasonable to rely exclusively on the financial market assessments in order to assess a situation. Assessing the value of a financial security is not comparable to measuring an objective magnitude, like, for example, estimating the weight of an object. A financial security is a claim on future revenue: in order to evaluate it, one must anticipate what this future will be. It is a matter of appraisal, not of objective measure, because at the instant $\mathrm{t}$, the future is by no means predetermined. In trading rooms, it is what operators imagine it will be. A financial price is the result of an assessment, a belief, a bet on the future: there is no guarantee that the assessment of markets is in any way superior to other forms of assessment. Above all, financial evaluation is not neutral: it affects the object it is meant to measure, it initiates and builds the future it imagines. So, rating agencies play an important role in determining interest rates on bond markets by awarding grades that are highly subjective, if they are not driven by a desire to fuel instability, a source for speculative profits. When agencies degrade the rating of a state, they increase the rate of interest demanded by financial actors in order to acquire securities of the public debt of this state, and thereby increase the risk of bankruptcy they have announced.

According to the supporters of the theory of efficient financial markets, the operators objectively analyse the conditions of public finance in order to evaluate the risk of acquiring government bonds. Let us take the case of the Greek deficit: the financial operators and governors based themselves exclusively on evaluations of a financial type to judge the risks of government bonds. Thus, when the interest rate required to finance the Greek deficit increased up to over $10 \%$, they all concluded that there was a high risk of default (bankruptcy): if investors were asking for such a high risk premium, then the danger must have been extreme.

However, this is a serious error if we consider the effective nature of the evaluation of the financial market. Not being efficient, markets quite often determine prices that are completely disconnected from the fundamental values. In these circumstances, it is unreasonable to trust solely in the estimate of the financial market to evaluate the situation. Estimating the value of a share of stock or a bond is in fact not comparable to that of measuring an objective magnitude such as, for example, the weight of an object. A financial object is a right to that object's future earnings: in order to evaluate it, it is necessary to predict those earnings. This is a subjective evaluation, not an objective measurement, because at instant $t$, the future is in no way predetermined. The evaluation is based on what the financial operators expect for that future. The price of a stock or bond is the result of a prediction, a belief, a gamble: there is no guarantee that the judgment of the market is in any way better than any other form of judgment.

In particular, financial evaluation is not neutral; it affects the object that it is meant to measure, contributing to the creation of the future that it has imagined. Thus, the agencies that evaluate financial risks contribute in large measure to determining the interest rates in the bond markets, assigning evaluations that are strongly subjective, driven by the desire to feed instability, the source of speculative profits. When the rating agencies lower a country's rating, they increase the interest rate demanded by investors for acquiring bonds against public debt and 
thus increase the risk of bankruptcy that they themselves have declared.

\section{Appendix: On statistical models for the analysis of tests}

The use of multiple-choice tests is by now widespread in Italy as in other countries: for admission to university; for aptitude tests in schools; for admission to active teacher training programs, and so forth. These are often presented as efficient instruments for measuring the students' preparation.

The word measurement evokes the objectivity of measurements in physics, such as weight or height. To define fundamental measurements like these, it is necessary to concatenate the measurable objects in such a way that the measurement of the concatenation of A with B is the sum of the measurement of A and the measurement of B. All the measurements of physics can be derived from a small number of fundamental measurements.

In the social and educational sciences there are no fundamental measurements and thus there are no derived measurements either, because there is no reasonable way to define the operation of concatenation. Modern measurement theory admits, however, the possibility of defining the implicit measurement by means of the use of probabilistic models.

For example, in a multiple-choice test, if we want to measure the difficulty of the questions, it is not sufficient to count the number of students who answered the questions correctly, since this number also depends on the students' ability. It is thus necessary to find a way to separate the difficulty of the questions from the students' ability. This is possibly by hypothesising a probabilistic model that ties the rough data of the correct answers to the difficulty $\delta_{i}$ of the questions and to the ability $\beta_{j}$ of the students. The simplest of such models, the Rasch model, hypothesises the probability that a student of ability $\beta_{j}$ will correctly answer a question of difficulty $\delta_{i}$ is:

$p=e^{(\beta j-\delta i)} /\left(1+e^{(\beta j-\delta i)}\right)$.

Beginning with a model such as this, we can estimate the difficulty of the questions and the ability of the students. If the model hypothesised is in good agreement with the data, the quantity estimated can represent significant measurements; otherwise they are meaningless. Further, even if the model is in good agreement with the data, the measurements are estimates with an uncertainty that is generally much larger than what we are accustomed to dealing with in the direct measurements of physics.

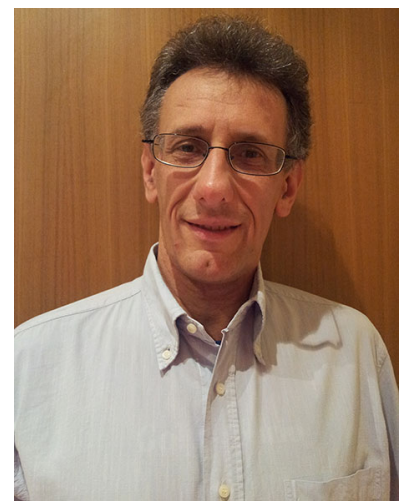

Enrico Rogora teaches mathematics at the Sapienza Università di Roma. After 10 years of work in the field of algebraic geometry and invariant theory, his main interests are now in the history of italian mathematics and in psychometry. 